Review Article

\title{
Prevalence of Escherichia coli in Under-Five Children with Diarrhea in Ethiopia: A Systematic Review and Meta-Analysis
}

\author{
Tizazu Zenebe $\mathbb{D}^{\mathrm{D}},{ }^{1}$ Meseret Mitiku, ${ }^{2}$ and Yonas Alem $^{3}$ \\ ${ }^{1}$ Department of Medicine, Medical Microbiology Unit, DebreBerhan University, DebreBerhan, Ethiopia \\ ${ }^{2}$ Department of Medical Laboratory Sciences, MaddaWalabu University, Bale Robe, Ethiopia \\ ${ }^{3}$ Department of Medical Laboratory Sciences, Ambo University, Ambo, Ethiopia \\ Correspondence should be addressed to Tizazu Zenebe; tizazuzenebe@yahoo.com
}

Received 8 June 2020; Accepted 26 August 2020; Published 7 September 2020

Academic Editor: Luigi Santacroce

Copyright ( 92020 Tizazu Zenebe et al. This is an open access article distributed under the Creative Commons Attribution License, which permits unrestricted use, distribution, and reproduction in any medium, provided the original work is properly cited.

Diarrhea remains as a high health burden, especially to children in low-income countries including Ethiopia. Diarrheagenic Escherichia coli have been commonly associated as bacterial pathogens causing diarrheal disease among children. This systemic review and meta-analysis was intended to determine the pooled prevalence of Escherichia coli in under-five children with diarrhea in Ethiopia. A comprehensive search in PubMed, Google Scholar, ScienceDirect, ResearchGate, and Google search engine and manual searching were done for this systematic review and meta-analysis. The eligibility criteria for selecting studies were studies involving under-five children with diarrhea in Ethiopia, published articles, cross-sectional studies, and articles reported in English. The study was conducted based on the Preferred Reporting Items for Systematic Reviews and Meta-Analysis (PRISMA) checklist. The data analysis was done using STATA 16.0 software. Cochran's Q-test and $I^{2}$ statistics were used for the assessment of heterogeneity. The random-effect model was used to estimate the pooled prevalence of Escherichia coli. A total of 797 articles were initially retrieved, and finally, 11 studies met the eligibility criteria and were included in the final meta-analysis. The pooled prevalence of Escherichia coli was 25\% (95\% CI: 9, 41). The pooled prevalence was varied by region, detection method, and sample size. The high prevalence emphasizes that Escherichia coli is a potential pathogen in under-five children with diarrhea in Ethiopia.

\section{Introduction}

The World Health Organization (WHO) defined diarrhea as the passage of three or more loose or liquid stools per day [1]. Due to the complex interplay of the environment, food, water, and sanitation with poverty and deprivation, diarrhea remains as a high health burden, especially to children in the world [2]. Diarrhea was one of the top ten causes of death, ninth globally, sixth in lower-middle-income countries, and the second in low-income countries in 2016 [3]. Ethiopia ranks sixth with the highest burden of pneumonia and diarrhea in the world [4]. National data [5], many local studies [6-10], and other reports [11] showed that diarrhea is still a common health problem in the country.

Diarrhea could be caused by different enteric pathogens which include bacteria, viruses, and parasites [12]. Diarrheagenic Escherichia coli, Campylobacter, Shigella, Vibrio cholerae, Salmonella, and Clostridium difficile are the common bacterial agents of diarrhea [12, 13]. Diarrheagenic $E$. coli have been predominantly associated as bacterial pathogens causing diarrheal disease among children in developing countries [14]. There are many local studies done on enteropathogens that showed bacterial pathogens causing diarrhea in under-five children in Ethiopia [15-17]. There are also studies done on E. coli in under-five children in the country [18-23]. However, there are no data on the pooled prevalence of E. coli at the national level in underfive children in Ethiopia. Therefore, this systemic review and meta-analysis was intended to determine the pooled prevalence of E. coli in under-five children in Ethiopia. And the comprehensive evidence will not only have an impact on control and prevention but also indicates the importance of the pathogens and the need of further studies in Ethiopia. 


\section{Methods}

2.1. Study Design. A systematic review and meta-analysis was conducted to estimate the pooled prevalence of $E$. coli in under-five children with diarrhea in Ethiopia. The study followed a similar approach with Alebe et al. [24] and was conducted based on the PRISMA checklist [25].

2.2. Literature Search Strategies for Relevant Studies. A comprehensive search with no date limits was performed in the following databases: PubMed, Google Scholar, ScienceDirect, ResearchGate, and Google search engine, and by manual searching. The search of the literature was conducted between March 1 and April 30, 2020. All articles published until April 30, 2020, were considered for conducting the review. E. coli, Under-five Children, Diarrhea, diarrhoea, and Ethiopia were used as search arm. We use search terms using Boolean operators for PubMed as follows: ((“"Escherichia coli”) AND (Children)) AND ("Diarrhea" OR "diarrhoea")) AND (Ethiopia) were used for searching. We also use Boolean operators for Google Scholar and ScienceDirect as follows: "Escherichia coli" and "Under-five" and "Children" and "diarrhea" or "diarrhoea" and "Ethiopia."

2.3. Study Eligibility Criteria. All available studies and data were incorporated based on the following predefined eligibility criteria. The inclusion criteria were studies conducted in under-five children with diarrhea in Ethiopia, published articles, cross-sectional study, and articles reported in English. The exclusion criteria were articles with duplicate or overlapping data and without full text available.

2.4. Study Selection. Records identified from various sources with the search strategies were exported to Endnote reference software version 7. Duplicate records were identified, recorded, and removed with Endnote and manually. For this, two authors independently screened the title and abstracts with the predefined inclusion criteria. Two authors also independently collected full texts and evaluated the eligibility of studies for final inclusion. When discrepancies between two authors occur, the other reviewers solve it through discussion and consensus.

2.5. Measurement of Outcome Variables. The outcome of the study was the pooled prevalence of $E$. coli in under-five children with diarrhea. The prevalence was calculated by dividing the numbers of bacterial isolates by the total number of clinically related patients. In other words, $E$. coli isolation rate was defined as the ratio of the number of positive samples for bacteria to the total number of sample size (negative and positive for bacteria).

2.6. Data Extraction. Data from selected articles were extracted using a standardized data extraction format, by two authors independently extracting all necessary data. Any disagreement during the data extraction was resolved through discussion and consensus. The primary author of the original research was contacted for additional information or to clarify method details as needed. The data extraction format included primary author, publication year, region, study area, sample size, detection method, and prevalence of E. coli.

2.7. Quality Assessment. Two authors independently assessed the risk of bias for each original study. The quality of the study was evaluated according to the Newcastle-Ottawa scale adapted for cross-sectional studies [26] and graded out of 10 points (10 stars). The tool has three major sections: methodological quality (with 5 stars), comparability of the study (with 2 stars), and outcomes related to statistical analysis (with 3 stars). The mean score of two authors with a third author negotiation was taken for final decision, and studies with a score greater than or equal to five were included.

2.8. Data Processing and Analysis. The relevant data were extracted from selected studies using format prepared in Microsoft Excel. The data analysis was done using STATA 16.0 software. The data entered into Microsoft excel were imported to the STATA for outcome measures and subgroup analyses. For the determination of variation in true effect sizes across population (clinical heterogeneity), a restricted maximumlikelihood random-effect model was applied. The original articles were described using forest plot, funnel plot, and tables. The random-effect model was used to compute the pooled prevalence of Escherichia coli. The estimated pooled prevalence with $95 \%$ confidence intervals by forest plot and publication bias by funnel plot were presented.

Subgroup analysis was performed based on region, publication year, detection method, and sample size. Studies from Addis Ababa City Administration, Tigray, and Amhara regional state were included, and a study from other regions was not available. The publication year was categorized into three: 1970-1990 (two decades), 1991-2010 (two decades), and 2011-2020 (a decade). The detection methods were grouped as follows: culture only, serotyping, serotyping and enterotoxin test, and molecular method.

Heterogeneity among reported prevalence was assessed by computing $p$ values of Cochran's $Q$-test and $I^{2}$ statistics [27]. Cochran's $Q$-test evaluates the existence of heterogeneity, and $p<0.10$ indicates statistically significant heterogeneity [28]. The $I^{2}$ statistics provides an estimate of the percentage of the variability in effect estimates that is due to heterogeneity rather than sampling error or chance differences. $I^{2}$ values of $25 \%$, $50 \%$, and $75 \%$ are considered to represent low, medium, and high heterogeneity, respectively $[27,28]$. Begg's rank test and Egger's regression test are among various statistical tests used for checking publication bias in the funnel plot [29]. Begg's rank test examines the correlation between the effect sizes and their corresponding sampling variances. And a strong correlation implies publication bias. Egger's test regresses the standardized effect sizes on their precisions; in the absence of publication bias, the regression intercept is expected to be zero [29]. Egger's test at 5\% significant level was done to check publication bias [29, 30]. 


\section{Results}

A total of 797 articles were initially retrieved for the prevalence of $E$. coli in under-five children with diarrhea using the range of databases described above. Among the total, 351 articles were removed after duplicates, and again after screening, 324 articles were excluded. Moreover, 108 articles were excluded based on title and abstract, involving nonhuman and adult participants, inaccessibility of full texts, and studies conducted in other countries. And also, 4 more articles were excluded due to insufficient information. Finally, 10 studies met the eligibility criteria and were included in the final meta-analysis (Figure 1).

3.1. Description of Search Results. All the included 10 articles (Table 1) used a cross-sectional study design and were published from 1976 to 2019, data in the last 4 and more decades. A total of 2269 (ranging from 98 to 422) study participants were involved to determine the pooled prevalence of $E$. coli among under-five children with diarrhea. These studies were conducted in Addis Ababa City Administration and Amhara regional state, Ethiopia. Majority of the studies (8 studies) were done in Addis Ababa $[19,21-23,31-33]$, and the remaining 2 were done in Amhara regional state $[17,18]$. Three of the studies $[21,22,31]$ were very old (published before 2000) compared to the others. The lowest prevalence was 20.8\% [19] and the highest prevalence was $62.7 \%$ [17]. There were no reported data from the other regional states and city administration in Ethiopia during the preparation of the present review.

\subsection{Prevalence of Escherichia coli in Under-Five Children.} In the present systemic and meta-analysis, the pooled prevalence of E. coli was $29 \%$ (95\% CI: 10, 48). There was no heterogeneity observed across the included studies $\left(I^{2}=0.00 \% ; Q=1.84, p=1.00\right)$. Regardless of the absence of heterogeneity, the random-effect model was used to estimate the pooled prevalence of $E$. coli in under-five children with diarrhea in Ethiopia (Figure 2). In this meta-analysis, the lowest prevalence was $20.8 \%$ [19] and the highest prevalence was $62.7 \%$ [17]. To check for the presence of publication bias, funnel plot asymmetry was used (Figure 3). The funnel plot showed asymmetry, which was indicative of the presence of publication bias. However, Egger's tests showed that there was no statistically significant publication bias in estimating the prevalence of $E$. coli in under-five children $(p=0.2611)$.

3.3. Subgroup Analysis. A subgroup analysis for region, publication year, method of detection, and sample size was done. A higher estimate of the pooled prevalence of $E$. coli (54, 95\% CI: -0.21, 1.29) was observed in Amhara regional state (Table 2). Almost similar estimate of the pooled prevalence of $E$. coli $(21 \%)$ was observed across the two last decades. A higher prevalence of E. coli was detected through serotyping and enterotoxin test (31\% with 95\% CI: $-12,75)$. Studies with sample size greater than 200 have higher estimated pooled prevalence (26\% (95\% CI: 2, 49)) compared to those with sample size less than or equal to 200 .

\section{Discussions}

E. coli are not only important member of the normal intestinal microbiota of humans and other mammals but also they are highly specialized pathogenic strains causing worldwide outbreaks of severe diseases and opportunistic pathogens $[34,35]$. Mainly there are six intestinal pathotypes $[34,36]$. These are enteropathogenic E. coli, Shiga toxinproducing $E$. coli, enterotoxigenic $E$. coli, enteroinvasive $E$. coli, enteroaggregative $E$. coli, and diffusely adherent E. coli. However, identifying such pathogenic or diarrheagenic E. coli strains needs molecular techniques such as polymerase chain reaction (PCR) [36]. In the present systemic and meta-analysis, majority of the included studies used conventional methods for the detection of $E$. coli. This means that the reported prevalence of E. coli in these studies may not be necessarily true pathogen or diarrheagenic E. coli. A bacteriological analysis shows that 100 water samples (50 from cases and 50 from controls) were found to be positive for total coliforms [37]. In the study, $70 \%$ of sampled water from cases and $74 \%$ of sampled water from controls were positive for E. coli [37]. This supports that $E$. coli may not be necessarily the cause of the disease case (diarrhea) unless confirmed by advanced laboratory techniques.

The estimated pooled prevalence of $E$. coli was $25 \%$ in the present study which could be considered as a higher prevalence rate. Except the two studies $[23,31]$, all were done with conventional detection methods that could contribute to the higher prevalence of the bacteria (nonpathogenic versus pathogenic). The present pooled prevalence of $E$. coli was higher compared to the pooled prevalence of Shigella species (6.6\%) reported in systemic review and meta-analysis done in Ethiopia [38]. In a systemic and meta-analysis study done in foods of animal origin (beef, milk, mutton, chevon, and chicken) and environmental swabs in Ethiopia, the estimated pooled prevalence of E. coli was 15\% [39] which is less than that reported in the present study. On the contrary, meta-analysis study done on E. coli O157: $\mathrm{H} 7$ found 4\% prevalence in foods of animal origin [40] and 5\% pooled prevalence of E. coli O157 : H7 [41]. This discrepancy may be due to difference in study subjects, and detection method, or true prevalence.

In the subgroup analysis, the estimated pooled prevalence of $E$. coli was varied by region, sample size, and detection method. All studies were done in Addis Ababa City Administration and Amhara regional state. The estimated pooled prevalence is relatively higher in Amhara regional state (54\%) than in Addis Ababa (27\%). It may be due to difference in access to improved source of drinking water, $97 \%$ access to urban households and $57 \%$ to rural households [5]. And also difference in essential hand-washing agents, the availability of soap and water, is highest in Addis Ababa (39\%) and lowest in Amhara (5\%) [5]. In addition, difference in diagnosis method used, actual prevalence difference, geographical location, and difference in the 


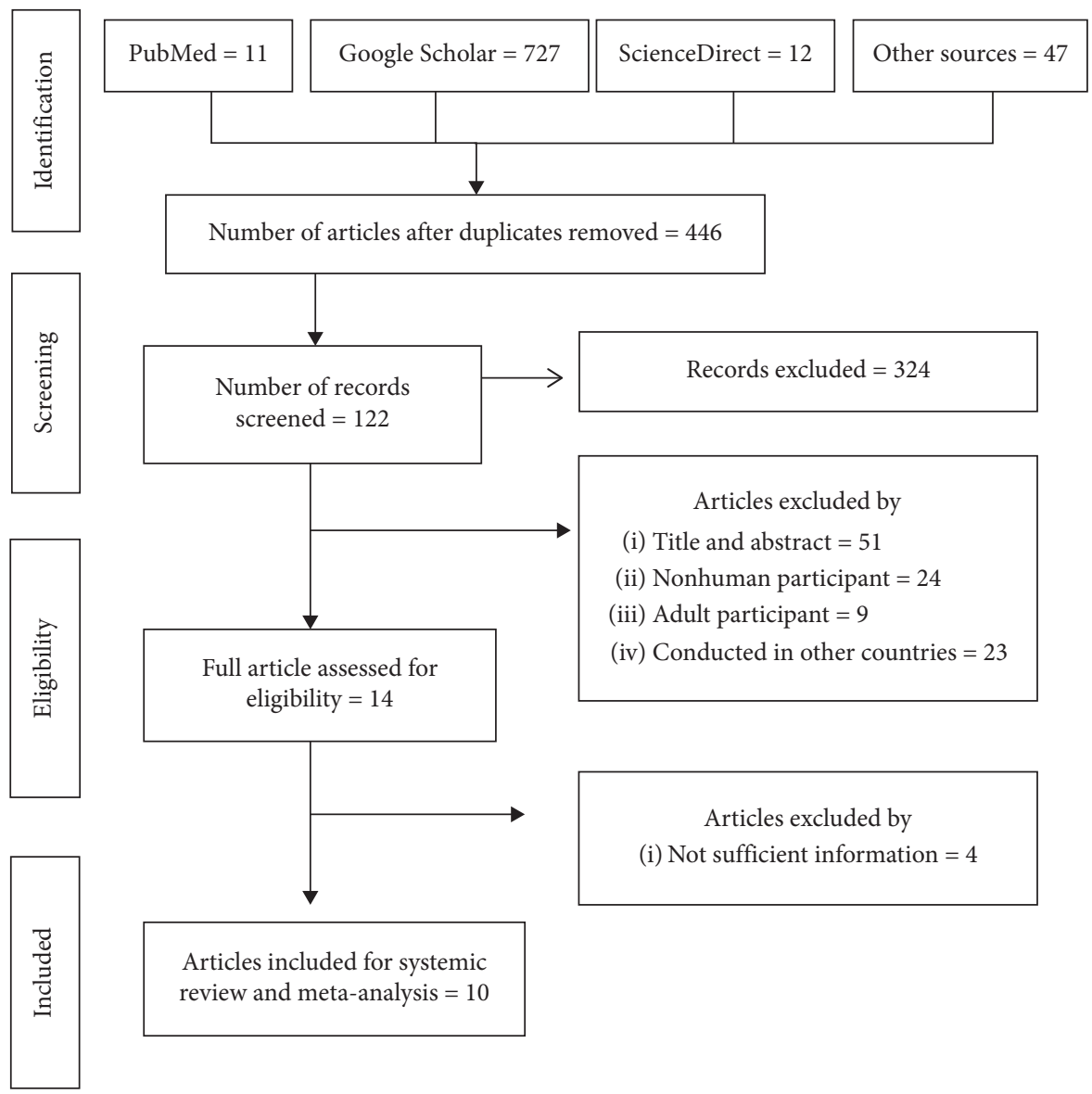

FIGURE 1: Flowchart of study selection for systematic review and meta-analysis of the prevalence of Escherichia coli in under-five children with diarrhea in Ethiopia.

Table 1: Summary of selected studies reporting the prevalence of Escherichia coli in different parts of Ethiopia, from 1970 to 2019.

\begin{tabular}{|c|c|c|c|c|c|c|c|}
\hline Study & $\begin{array}{l}\text { Quality } \\
\text { score }\end{array}$ & $\begin{array}{c}\text { Publication } \\
\text { year }\end{array}$ & Region & Study area & $\begin{array}{l}\text { Sample } \\
\text { size }\end{array}$ & Detection method & $\begin{array}{c}\text { Prevalence of E. coli } \\
(\%)\end{array}$ \\
\hline $\begin{array}{l}\text { Wadstrom et al. } \\
\text { [22] }\end{array}$ & 7 & 1976 & \multirow{8}{*}{$\begin{array}{l}\text { Addis } \\
\text { Ababa }\end{array}$} & $\begin{array}{l}\text { Addis } \\
\text { Ababa }\end{array}$ & 354 & $\begin{array}{l}\text { Serotyping and } \\
\text { enterotoxin test }\end{array}$ & 37 \\
\hline Thoren et al. [21] & 6 & 1982 & & $\begin{array}{l}\text { Addis } \\
\text { Ababa }\end{array}$ & 175 & $\begin{array}{l}\text { Serotyping and } \\
\text { enterotoxin test }\end{array}$ & 28 \\
\hline Geyid et al. [31] & 5 & 1998 & & $\begin{array}{l}\text { Addis } \\
\text { Ababa }\end{array}$ & 108 & DNA probe & 58 \\
\hline Asrat [19] & 5 & 2001 & & $\begin{array}{l}\text { Addis } \\
\text { Ababa }\end{array}$ & 345 & Serotyping & 20.8 \\
\hline Mamuye et al. [32] & 5 & 2015 & & $\begin{array}{l}\text { Addis } \\
\text { Ababa }\end{array}$ & 253 & Culture & 24.1 \\
\hline Zenebe [23] & 5 & 2018 & & $\begin{array}{l}\text { Addis } \\
\text { Ababa }\end{array}$ & 98 & Culture & 59.2 \\
\hline Zenebe [23] & 5 & 2018 & & $\begin{array}{l}\text { Addis } \\
\text { Ababa }\end{array}$ & 98 & Culture & 25.5 \\
\hline $\begin{array}{l}\text { GebreSilasie et al. } \\
\text { [33] }\end{array}$ & 5 & 2018 & & $\begin{array}{l}\text { Addis } \\
\text { Ababa }\end{array}$ & 253 & Culture & 24.1 \\
\hline Zelelie et al. [17] & 5 & 2019 & \multirow[t]{2}{*}{ Amhara } & $\begin{array}{l}\text { Debre } \\
\text { Berhan }\end{array}$ & 163 & Culture & 62.7 \\
\hline Adugna et al. [18] & 6 & 2015 & & Bahr Dar & 422 & Serotyping & 48.3 \\
\hline
\end{tabular}

number of studies involved in the analysis could make the difference. Still the prevalence in Addis Ababa is higher (27\%). This may be due to the sanitation problem according to a report by Adane et al. [6] which indicated that $94.6 \%$ of unimproved sanitation facilities and greater intermittent water supply [42] occurred in Addis Ababa. In both study 


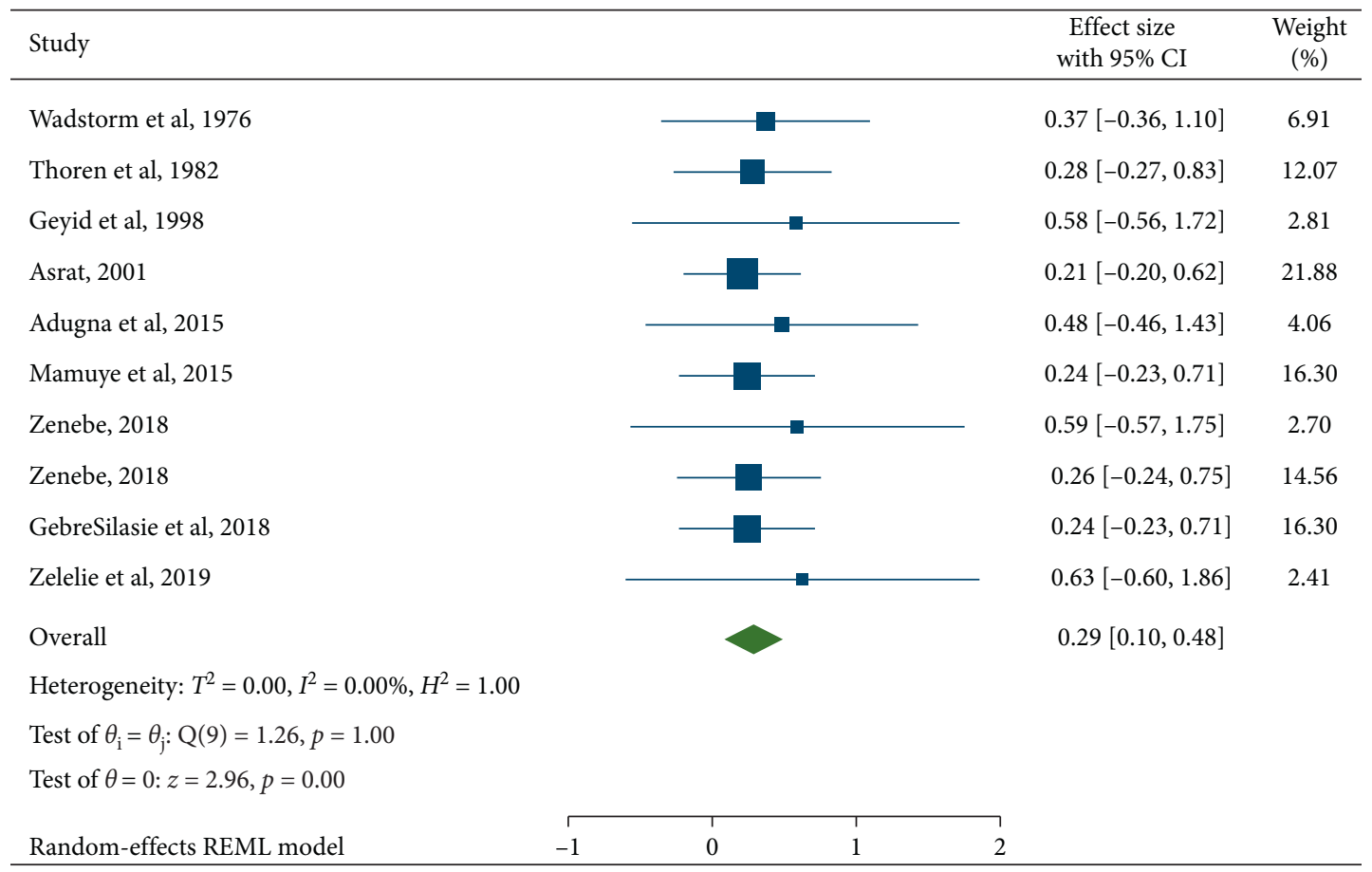

FIgURE 2: Forest plot of the pooled prevalence of Escherichia coli in under-five children with diarrhea in Ethiopia.

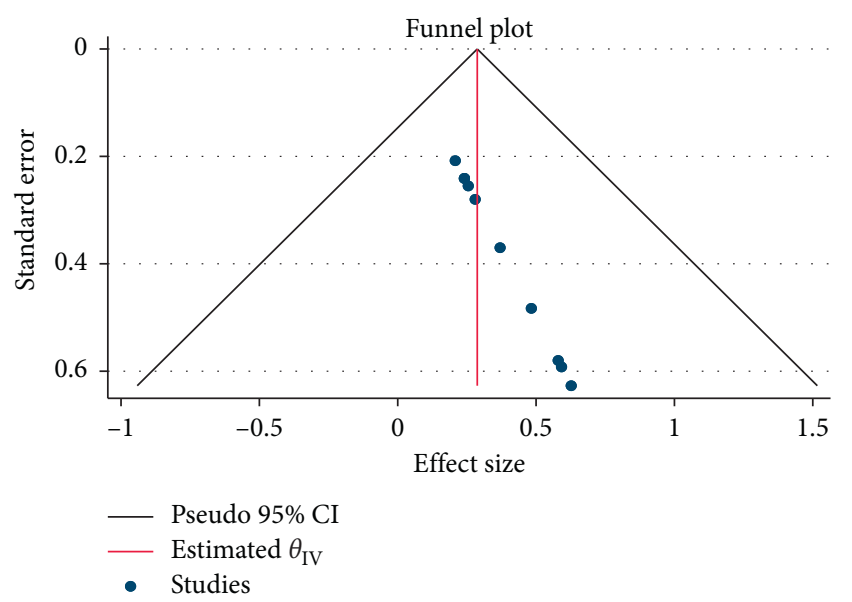

Figure 3: Funnel plot with $95 \%$ confidence limits of the pooled prevalence of Escherichia coli in under-five children with diarrhea in Ethiopia.

areas, the higher prevalence signifies the need for molecular characterization of pathogenic strains for a better understanding of prevention and control strategies.

The prevalence of $E$. coli is almost unchanged through time (over 43 years). It may be indicative of the absence or lack of interventions against the infection of E. coli or it may be neglected among common communicable disease-causing agents. In terms of detection methods, the higher prevalence was seen by serotyping and enterotoxin test $(31 \%)$. In contrary to the reality, the lower prevalence was reported using the culture detection method (19\%). According to a report by Zenebe [23], the prevalence of
E. coli was $59.2 \%$ using the culture method, but with PCR it was $25.5 \%$ which is expected. Thus, the inconsistency might be due to the number of studies included in the analysis for each method. The estimated pooled prevalence of $E$. coli was $35 \%$ for sample size equal to or less than 200 which is relatively higher than for sample size greater than 200 (26\%) but not statically significant.

The main limitation of the present study is that majority of the studies were done using the culture detection method only which could not rule out pathogenic E. coli from nonpathogenic. In addition, few numbers of articles were included mainly due to less number of studies done in 
TABLE 2: Subgroup estimate of the pooled prevalence of Escherichia coli in under-five children with diarrhea in Ethiopia.

\begin{tabular}{lcccc}
\hline Variables & Characteristics & Included studies & Sample size & Prevalence of E. coli (95\% CI) \\
\hline \multirow{2}{*}{ Region } & Addis Ababa & 8 & 1684 & $27(7,47)$ \\
& Amhara & 2 & 585 & $54(-0.21,1.29)$ \\
\hline \multirow{2}{*}{ Publication year } & $1976-1990$ & 3 & 637 & $35(-6,76)$ \\
& $1991-2010$ & 1 & 345 & $21(-20,62)$ \\
\hline \multirow{3}{*}{ Detection method } & $2011-2019$ & 6 & 1356 & $21(3,39)$ \\
& Serotyping & 2 & 767 & $25(-12,63)$ \\
& Serotyping and enterotoxin test & 2 & 529 & $31(-12,75)$ \\
By sample size & Culture only & 5 & 934 & $19(0,38)$ \\
& Molecular method & 2 & 206 & $31(-15,77)$ \\
\hline
\end{tabular}

Ethiopia. And also, the absence of data from other regional states and city administration could be considered as another potential limitation of the study.

\section{Conclusions}

Regardless of differentiating and assuring the pathogenic from nonpathogenic, a high prevalence of E. coli was observed in under-five children with diarrhea in Ethiopia. The study highlighted that E. coli may be considered as a potential pathogen in under-five children with diarrhea in Ethiopia. The finding justifies the need for molecular characterization and further epidemiological studies which might be important for searching preventive and intervention strategies. And also, generating data from other regional states and city administration could be useful for a full-blown picture of the problem at a country level.

\section{Data Availability}

The datasets used and/or analyzed during the current study are available from the corresponding author on reasonable request.

\section{Conflicts of Interest}

The authors declare that they have no conflicts of interest.

\section{References}

[1] WHO, Diarrhoeal disease, WHO, Geneva, Switzerland, 2017.

[2] GBD, "Estimates of global, regional, and national morbidity, mortality, and aetiologies of diarrhoeal diseases: a systematic analysis for the Global Burden of Disease Study 2015," The Lancet Infectious Diseases, vol. 17, no. 9, pp. 909-948, 2017.

[3] WHO, The Top 10 Causes of Death, World Health Organization, Geneva, Switzerland, 2018.

[4] IVAC, Pneumonia Progress Report, IVAC, Dhaka, Bangladesh, 2017.

[5] CSA, Ethiopia Demographic and Health Survey 2016, CSA, Ethiopia Mini Demographic and Health Survey 2016, pp. 1551, CSA, Addis Ababa, Ethiopia, 2016.

[6] A. Metadel, M. Bezatu, K. Helmut, M. Girmay, and W. Mulat, "Sanitation facilities, hygienic conditions, and prevalence of acute diarrhea among under- five children in slums of Addis
Ababa, Ethiopia: baseline survey of a longitudinal study," PLoS One, vol. 12, no. 8, pp. 1-18, Article ID e0182783, 2017.

[7] Z. A. Anteneh, K. Andargie, and M. Tarekegn, "Prevalence and determinants of acute diarrhea among children younger than five years old in Jabithennan District, Northwest Ethiopia, 2014," BMC Public Health, vol. 17, no. 99, 2017.

[8] H. Bizuneh, F. Getnet, B. Meressa, Y. Tegene, and G. Worku, "Factors associated with diarrheal morbidity among underfive children in Jigjiga town, Somali Regional State, eastern Ethiopia: a cross-sectional study," BMC Pediatrics, vol. 17, no. $182,2017$.

[9] G. Gedamu, "Magnitude and associated factors of diarrhea among under five children in farta wereda, North West Ethiopia," Quality in Primary Care, vol. 25, no. 4, pp. 199-207, 2017.

[10] A. Tamiso, M. Yitayal, and A. Awoke, "Prevalence and determinants of childhood diarrhoea among graduated households, in rural area of shebedino District, southern Ethiopia, 2013," Science Journal of Public Health, vol. 2, no. 3 , pp. 243-251, 2014.

[11] CDC, Global Heath-Ethiopia, CDC in Ethiopia Factsheeet, CDC, Atlanta, GA, USA, 2017.

[12] K. Hodges and R. Gill, "Infectious diarrhea," Gut Microbes, vol. 1, no. 1, pp. 4-12, 2010.

[13] M. Farthing, M. A. Salam, G. Lindberg et al., "Acute diarrhea in adults and children," Journal of Clinical Gastroenterology, vol. 47, no. 1, pp. 12-20, 2013.

[14] K. L. Kotloff, "The burden and etiology of diarrheal illness in developing countries," Pediatric Clinics of North America, vol. 64, no. 4, pp. 799-814, 2017.

[15] G. Ameya, T. Tsalla, F. Getu, and E. Getu, "Antimicrobial susceptibility pattern, and associated factors of Salmonella and Shigella infections among under five children in Arba Minch, South Ethiopia," Annals of Clinical Microbiology and Antimicrobials, vol. 17, no. 1, 2018.

[16] M. Getamesay, B. Getenet, and Z. Ahmed, "Prevalence of Shigella, Salmonella and cmpylobacter species and their susceptibility patters among under five children with diarrhea in Hawassa town, south Ethiopia," Ethiopian Journal of Health Sciences, vol. 24, no. 2, pp. 101-108, 2014.

[17] T. Z. Zelelie, D. S. Gebreyes, A. T. Tilahun, H. A. Craddock, and N. Z. Gishen, "Enteropathogens in under-five children with diarrhea in health facilities of Debre Berhan Town, North Shoa, Ethiopia," Ethiopian Journal of Health Sciences, vol. 29, no. 2, pp. 203-214, 2019.

[18] A. Adugna, M. Kibret, B. Abera, E. Nibret, and M. Adal, "Antibiogram of E. coli serotypes isolated from children aged 
under five with acute diarrhea in Bahir Dar town," African Health Sciences, vol. 15, no. 2, pp. 656-663, 2015.

[19] D. Asrtat, "Screening for enteropathogenic Escherchia coli (EPEC) in paedatrics patients with diarrhea and control using pooled," Antisera, vol. 39, pp. 23-27, 2001.

[20] G. Stintzing and R. Möllby, "Colonization of the upper jejunum by enteropathogenic and enterotoxigenic Escherichia coli in paediatric diarrhoea," Acta Paediatrica, vol. 71, no. 3, pp. 457-465, 1982.

[21] A. Thoren, G. Stintzing, B. Tufvesson, M. Walder, and D. Habte, "Aetiology and clinical features of severe infantile diarrhoea in Addis Ababa, Ethiopia," Journal of Tropical Pediatrics, vol. 28, no. 3, pp. 127-131, 1982.

[22] T. Wadistrom, A. Aust-Ketis, D. Habte et al., "Enterotoxinproducing bacteria and parasites in stools of Ethiopian children with diarrhoeal disease," Archives of Disease in Childhood, vol. 51, no. 11, pp. 865-870, 1976.

[23] B. Zenebe, Pathogenic Escherichia coli strains and their antibiotic susceptibility profiles in cases of child diarrhea at tikur anbessa general specialized Hospital, AAU College of Health Sciences, Addis Ababa, Ethiopia, 2018.

[24] A. Alebel, C. Tesema, B. Temesgen, A. Gebrie, P. Petrucka, and G. D. Kibret, "Prevalence and determinants of diarrhea among under-five children in Ethiopia: a systematic review and meta-analysis," PLoS One, vol. 13, no. 6, Article ID e0199684, 2018.

[25] D. Moher, A. Liberati, J. Tetzlaff, and D. G. Altman, "Preferred reporting items for systematic reviews and meta-analyses: the PRISMA statement," BMJ, vol. 339, 2009.

[26] O. Newcastle, "Newcastle- Ottawa: scale customized for crosssectional studies in," 2018, https://static-contentspringer. com/esm//12889_2012_5111_MOESM3_ESMdoc.

[27] G. Rücker, G. Schwarzer, J. R. Carpenter, and M. Schumacher, "Undue reliance on $\mathrm{I} 2$ in assessing heterogeneity may mislead," BMC Medical Research Methodology, vol. 8, no. 79, 2008.

[28] J. P. Ioannidis, "Interpretation of tests of heterogeneity and bias in meta-analysis," Journal of Evaluation in Clinical Practice, vol. 14, pp. 1356-2294, 2008.

[29] L. Lin and H. Chu, "Quantifying publication bias in metaanalysis," Biometrics, vol. 74, no. 3, pp. 785-794, 2018.

[30] M. Borenstein, L. V. Hedges, J. P. T. Higgins, and H. R. Rothstein, "A basic introduction to fixed-effect and random-effects models for meta-analysis," Research Synthesis Methods, vol. 1, no. 2, pp. 97-111, 2010.

[31] A. Geyid, O. Olsvik, and A. Ljungh, "Virulence properties of Escherchia coli isolated from Ethiopian patients with acute or persitent diarrhea," Ethiopian Medical Journal, vol. 36, pp. 123-137, 1998.

[32] Y. Mamuye, G. Metaferia, A. Birhanu, K. Desta, and S. Fantaw, "Isolation and antibiotic susceptibility patterns of Shigella and Salmonella among under 5 children with acute diarrhoea: a cross-sectional study at selected public health facilities in Addis Ababa, Ethiopia," Clinical Microbiology: Open Access, vol. 4, no. 1, pp. 1-7, 2015.

[33] Y. M. GebreSilasie, K. D. Tullu, and A. G. Yeshanew, "Resistance pattern and maternal knowledge, attitude and practices of suspected Diarrheagenic Escherichia coli among children under 5 years of age in Addis Ababa, Ethiopia: cross sectional study," Antimicrobial Resistance and Infection Control, vol. 7, no. 10, pp. 1-9, 2018.

[34] C. Garcia-Aljaro and M. Momba, Pathogenic Members of Escherichia coli \& Shigella spp. shigellosis, UNESCO, London, UK, 2017.
[35] M. B. Martinez, W. Eliasb, I. C. Scaletskya, B. E. Gutha, J. F. Rodriguesc, and R. M. Piazza, "Diarrheagenic Escherichia coli," Brazilian Journal of Microbiology, vol. 47, pp. 3-30, 2016.

[36] M. A. Croxen, R. J. Law, R. Scholz, K. M. Keeney, and M. Wlodarska, "Recent advances in understanding enteric pathogenic Escherichia coli," Clinical Microbiology Reviews, vol. 26, no. 4, pp. 822-880, 2013.

[37] N. E. Finlay, S. R. Gari, A. B. Hailu, and B. M. Alemu, "Association between microbial water quality, sanitation and hygiene practices and childhood diarrhea in Kersa and Omo Nada districts of Jimma Zone, Ethiopia," PLoS One, vol. 15, no. 2, Article ID e0229303, 2020.

[38] S. Hussen, G. Mulatu, and Z. Y. Kassa, "Prevalence of Shigella species and its drug resistance pattern in Ethiopia: a systematic review and meta-analysis," Annals of Clinical Microbiology and Antimicrobials, vol. 18, no. 22, pp. 1-11, 2019.

[39] A. Assefa and A. Bihon, "A systematic review and metaanalysis of prevalence of Escherichia coli in foods of animal origin in Ethiopia," Heliyon, vol. 4, Article ID e00716, 2018.

[40] A. Assefa, "Prevalence of Escherichia coli O157:H7 in foods of animal origin in Ethiopia: a meta-analysis," Cogent Food \& Agriculture, vol. 5, no. 1, Article ID 1642981, 2019.

[41] A. Zelalem, M. Sisay, J. L. Vipham, K. Abegaz, A. Kebede, and Y. Terefe, "The prevalence and antimicrobial resistance profiles of bacterial isolates from meat and meat products in Ethiopia: a systematic review and meta-analysis," International Journal of Food Contamination, vol. 6, no. 1, pp. 1-14, 2019.

[42] M. Adane, B. Mengistie, G. Medhin, H. Kloos, and W. Mulat, "Piped water supply interruptions and acute diarrhea among under-five children in Addis Ababa slums, Ethiopia: a matched casecontrol study," PLoS One, vol. 12, no. 7, Article ID e0181516, 2017. 